\title{
Basicity of the System of Exponents with a Linear Phase in Sobolev Weight Space
}

\author{
Togrul Muradov and Valid Salmanov \\ Institute of Mathematics and Mechanics of NAS of Azerbaijan, B. Vahabzade Street 9, 1141 Baku, Azerbaijan \\ Correspondence should be addressed to Valid Salmanov; department2011@mail.ru
}

Received 28 May 2014; Accepted 12 August 2014; Published 1 September 2014

Academic Editor: Bruce A. Watson

Copyright (C) 2014 T. Muradov and V. Salmanov. This is an open access article distributed under the Creative Commons Attribution License, which permits unrestricted use, distribution, and reproduction in any medium, provided the original work is properly cited.

In the present paper a criterion for basicity of exponential system with linear phase is obtained in Sobolev weight space $W_{p, \rho}^{1}(-\pi, \pi)$.

In solving mathematical physics problems by the Fourier method, there often arise the systems of exponents of the form

$$
\begin{gathered}
\left\{e^{i(n t+\alpha(t))} ; e^{-i(n t+\beta(t))}\right\}_{n \geq 1}, \\
1 \cup\left\{e^{i(n t+\alpha(t))} ; e^{-i(n t+\beta(t))}\right\}_{n \geq 1},
\end{gathered}
$$

where $\alpha(t)$ and $\beta(t)$ are continuous or piecewise-continuous functions. Substantiation of the method requires studying the basis properties of these systems in Lebesgue and Sobolev spaces of functions. In the case when $\alpha(t)$ and $\beta(t)$ are linear functions, the basis properties of these systems in $L_{p}(-\pi, \pi)$, $1<p<+\infty$, were completely studied in the papers [19]. The weighted case of the space $L_{p}$ was considered in the papers $[10,11]$. The basis properties of these systems in Sobolev spaces were studied in [12-14]. It should be noted that the close problems were also considered in [15].

In the present paper we study basis properties of the systems (1) and (2) in Sobolev weight spaces when $\alpha(t)=\alpha t$, $\beta(t)=\alpha t$, where $\alpha$ is a real parameter. Therewith the issue of basicity of system (2) in Sobolev spaces is reduced to the issue of basicity of system (1) in respective Lebesgue spaces.

Let $L_{p, \rho}(-\pi, \pi)$ and $W_{p, \rho}^{1}(-\pi, \pi)$ be weight spaces with the norms

$$
\begin{aligned}
\|u\|_{L_{p, \rho}}^{p} & =\int_{-\pi}^{\pi}|u(\theta)|^{p} \rho(\theta) d \theta, \\
\|u\|_{W_{p, \rho}^{1}} & =\|u\|_{L_{p, \rho}}+\left\|u^{\prime}\right\|_{L_{p, \rho}},
\end{aligned}
$$

respectively, where $\rho(t)=\prod_{i=1}^{l}\left(\sin \left|\left(t-\tau_{i}\right) / 2\right|\right)^{\beta_{i}},-\pi<\tau_{1}<$ $\tau_{2}<\cdots<\tau_{l}<\pi$. Denote by $\mathscr{L}_{p, \rho}(-\pi, \pi)$ the direct sum $\mathscr{L}_{p, \rho}=L_{p, \rho} \oplus \mathbb{C}$, where $\mathbb{C}$ is the complex plane. The norm in this space is defined by the expression $\|\widehat{u}\|_{\mathscr{L}_{p, \rho}}=\|u\|_{L_{p, \rho}}+|\lambda|$, where $\widehat{u}=(u ; \lambda) \in \mathscr{L}_{p, \rho}$.

The following easily provable lemmas play an important role in obtaining the main results. It holds the following.

Lemma 1. Let $\beta_{i} \in(-1, p-1), i=\overline{1, l} ; p \in(1,+\infty)$. Then the operator

$$
A(u ; \lambda)=\lambda+\int_{-\pi}^{t} u(\theta) d \theta
$$

realizes an isomorphism between the spaces $\mathscr{L}_{p, \rho}$ and $W_{p, \rho}^{1}(-\pi, \pi)$; that is, the spaces $\mathscr{L}_{p, \rho}$ and $W_{p, \rho}^{1}$ are isomorphic.

Proof. At first prove the boundedness of the operator A. We have

$$
\begin{aligned}
\|A \hat{u}\|_{W_{p, \rho}^{1}} & =\left\|\lambda+\int_{-\pi}^{t} u(\theta) d \theta\right\|_{L_{p, \rho}}+\|u\|_{L_{p, \rho}} \\
& \leq\|\lambda\|_{L_{p, \rho}}+\left\|\int_{-\pi}^{t} u(\theta) d \theta\right\|_{L_{p, \rho}}+\|u\|_{L_{p, \rho}} \\
& =\left(\int_{-\pi}^{\pi}|\lambda|^{p} \rho(\theta) d \theta\right)^{1 / p}
\end{aligned}
$$




$$
\begin{aligned}
& +\left(\int_{-\pi}^{\pi}\left|\int_{-\pi}^{t} u(\theta) d \theta\right|^{p} \rho(t) d t\right)^{1 / p} \\
& +\|u\|_{L_{p, \rho}} \leq|\lambda|\|\rho\|_{L_{1}}^{1 / p} \\
& +\left(\int_{-\pi}^{\pi}\left(\int_{-\pi}^{t}|u(\theta)| d \theta\right)^{p} \rho(t) d t\right)^{1 / p} \\
& +\|u\|_{L_{p, \rho}} \\
& \leq|\lambda|\|\rho\|_{L_{1}}^{1 / p}+\left(\int_{-\pi}^{\pi}\left(\int_{-\pi}^{\pi}|u(\theta)| d \theta\right)^{p} \rho(t) d t\right)^{1 / p} \\
& +\|u\|_{L_{p, \rho}} \\
& =|\lambda|\|\rho\|_{L_{1}}^{1 / p}+\int_{-\pi}^{\pi}|u(\theta)| d \theta\|\rho\|_{L_{1}}^{1 / p}+\|u\|_{L_{p, \rho}} .
\end{aligned}
$$

Having applied the Holder inequality, hence we get

$$
\begin{aligned}
\int_{-\pi}^{\pi}|u(\theta)| d \theta & =\int_{-\pi}^{\pi}|u(\theta)| \rho^{1 / p} \rho^{-1 / p} d \theta \\
\leq & \left(\int_{-\pi}^{\pi}|u(\theta)|^{p} \rho d \theta\right)^{1 / p} \\
& \times\left(\int_{-\pi}^{\pi} \rho^{-q / p}(\theta) d \theta\right)^{1 / q} \\
= & \|u\|_{L_{p, \rho}}\left\|\rho^{1 /(1-p)}\right\|_{L_{1}}^{1 / q},
\end{aligned}
$$

where

$$
\frac{1}{p}+\frac{1}{q}=1
$$

Consequently

$$
\begin{aligned}
\|A \widehat{u}\|_{W_{p, \rho}^{1}} \leq & |\lambda|\|\rho\|_{L_{1}}^{1 / p}+\|u\|_{L_{p, \rho}}\|\rho\|_{L_{1}}^{1 / p}\left\|\rho^{1 /(1-p)}\right\|_{L_{1}}^{1 / q} \\
& +\|u\|_{L_{p, \rho}} \\
= & |\lambda| \rho_{L_{1}}^{1 / p}+\left(1+\|\rho\|_{L_{1}}^{1 / p}\left\|\rho^{1 /(1-p)}\right\|_{L_{1}}^{1 / q}+1\right)\|u\|_{L_{p, \rho}} \\
\leq & M\left(|\lambda|+\|u\|_{L_{p, \rho}}\right) \\
= & M\|\widehat{u}\|_{\mathscr{L}_{p, \rho}},
\end{aligned}
$$

where

$$
M=\max \left\{\|\rho\|_{L_{1}}^{1 / p} ;\|\rho\|_{L_{1}}^{1 / p}\left\|\rho^{1 /(1-p)}\right\|_{L_{1}}^{1 / q}+1\right\}
$$

Let us show that $\operatorname{Ker} A=\{0\}$. Put $A \widehat{u}=0$; that is,

$$
\lambda+\int_{-\pi}^{t} u(\theta) d \theta=0, \quad \forall t \in(-\pi, \pi),
$$

where $\lambda \in C, u \in \mathscr{L}_{p, \rho}$. By differentiating this equality, we get $u(\theta)=0$, a.e. on $(-\pi, \pi)$. Hence it follows that $\lambda=0$. From (11) it directly follows that $u=0$ a.e. on $(-\pi, \pi)$, and so $\widehat{u}=0$. Show that $\operatorname{Im} A=W_{p, \rho}^{1}(-\pi, \pi)(\operatorname{Im} A$ is the range of values of the operator $A)$. Let $v \in W_{p, \rho}^{1}(-\pi, \pi)$ be an arbitrary function. Let $\widehat{v}=\left(v^{\prime} ; v(0)\right)$. It is clear that $A \widehat{v}=v$ and $\widehat{v} \epsilon$ $\mathscr{L}_{p, \rho}$. Then from the Banach theorem we get that the operator $A$ is boundedly invertible.

The lemma is proved.

The following lemma is also valid.

Lemma 2. Let $p \in(1,+\infty)$ and $\beta_{i} \in(-1, p-1), i=\overline{1, l}$. Then for all $p_{0} \in(1, \alpha): L_{p, \rho}(-\pi, \pi) \subset L_{p_{0}}(-\pi, \pi)$, where

$$
\alpha=\min \left\{2 ; p ; \frac{p}{\beta_{1}+1} ; \frac{p}{\beta_{2}+1} ; \ldots ; \frac{p}{\beta_{l}+1}\right\} .
$$

Proof. Let $f \in L_{p, \rho}(-\pi, \pi), p_{0} \in(1, \alpha)$. We have

$$
\begin{aligned}
\int_{-\pi}^{\pi}|f|^{p_{0}} d t & \\
\quad & =\int_{-\pi}^{\pi}|f|^{p_{0}} \rho^{p_{0} / p} \rho^{-p_{0} / p} d t \\
& \leq\left(\int_{-\pi}^{\pi}|f|^{p} \rho d t\right)^{p_{0} / p}\left(\int_{-\pi}^{\pi} \rho^{p_{0} /\left(p_{0}-p\right)} d t\right)^{\left(p-p_{0}\right) / p} \\
& =\|f\|_{L_{p, \rho}}^{p_{0}}\left(\int_{-\pi}^{\pi} \prod_{i=1}^{l}\left(\sin \left|\frac{t-\tau_{i}}{2}\right|\right)^{\beta_{i} p_{0} /\left(p_{0}-p\right)} d t\right)^{\left(p-p_{0}\right) / p}
\end{aligned}
$$

Since $p_{0}<p /\left(\beta_{i}+1\right)$ and $\beta_{i}>-1$, then $\beta_{i} p_{0} /\left(p_{0}-p\right)>-1$, $i=\overline{1, l}$. It is easy to see that $f \in L_{p_{0}}(-\pi, \pi)$ and moreover $\|f\|_{L_{p_{0}}} \leq C\|f\|_{L_{p, \rho}}$.

The lemma is proved. lemma.

In obtaining the basic results we need the following main

Lemma 3. Let $f \in L_{p, \rho}(-\pi, \pi), \rho(t)=\prod_{i=1}^{l}\left(\sin \left|\left(t-\tau_{i}\right) / 2\right|\right)^{\beta_{i}}$ and $\beta_{i} \in(-1, p / q), i=\overline{1, l}, \alpha \in R$ be a real parameter, $1 / p+$ $1 / q=1$. Let $f$ have the expansion

$$
f(t)=\sum_{n \neq 0} c_{n} e^{i[n+\alpha \operatorname{sign} n] t}
$$

in the space $L_{p, \rho}(-\pi, \pi)$. Then it is valid

$$
\sum_{|n|>|\alpha|}\left|\frac{c_{n}}{n+\alpha \operatorname{sign} n}\right|<+\infty .
$$

Proof. As it follows from Lemma 2, $\exists p_{0} \in(1,2): L_{p, \rho} \subset$ $L_{p_{0}}$. At first consider the case when $\alpha>1 / 2 p_{0}-1,1 / p_{0}+$ $1 / q_{0}=1$. In this case the system $\left\{e^{i(n+\alpha \operatorname{sign} n) t}\right\}_{n \neq 0}$ is minimal in $L_{p_{0}}(0, \pi)$ (see [4]). Then from the results of the paper [16], 
the Hausdorff-Young inequality is valid for this system; that is,

$$
\left(\sum_{n \neq 0}^{\infty}\left|c_{n}\right|^{q_{0}}\right)^{1 / q_{0}} \leq M\|f\|_{L_{p_{0}}} .
$$

Applying the Holder inequality, we obtain

$$
\begin{aligned}
\sum_{|n|>|\alpha|}\left|\frac{c_{n}}{n+\alpha \operatorname{sign} n}\right| \leq & \left(\sum_{|n|>|\alpha|}\left|c_{n}\right|^{q_{0}}\right)^{1 / q_{0}} \\
& \times\left(\sum_{|n|>|\alpha|} \frac{1}{|n+\alpha \operatorname{sign} n|^{p_{0}}}\right)^{1 / p_{0}} .
\end{aligned}
$$

If $\alpha<1 / 2 p_{0}-1$, then $\exists k \in N, \alpha^{\prime}=\alpha+k>1 / 2 p_{0}-1$. Then

$$
\begin{aligned}
f(t) & =\sum_{n \neq 0} c_{n} e^{i(n+\alpha \operatorname{sign} n) t} \\
& =\sum_{n=-k}^{-1} c_{n} e^{i(n-\alpha) t}+\sum_{n=1}^{k} c_{n} e^{i(n+\alpha) t}+\sum_{|n| \geq k+1} c_{n} e^{i(n+\alpha \operatorname{sign} n) t} \\
& =f_{k}(t)+\sum_{n \neq 0} c_{n+k \operatorname{sign} n} e^{i(n+(k+\alpha) \operatorname{sign} n) t} \\
& =f_{k}(t)+\sum_{n \neq 0} c_{n+k \operatorname{sign} n} e^{i\left(n+\alpha^{\prime} \operatorname{sign} n\right) t}
\end{aligned}
$$

where $f_{k}(t)=\sum_{n=-k}^{-1} c_{n} e^{i(n-\alpha) t}+\sum_{n=1}^{k} c_{n} e^{i(n+\alpha) t}, \alpha^{\prime}=k+\alpha$. As a result, we have

$$
f(t)-f_{k}(t)=\sum_{n \neq 0} c_{n+k \operatorname{sign} n} e^{i\left(n+\alpha^{\prime} \operatorname{sign} n\right) t} .
$$

Since $\beta^{\prime}>1 / p_{0}-2$, then again from the results of the paper [16] it follows

$$
\left(\sum_{n \neq 0}\left|c_{n+k \operatorname{sign} n}\right|^{q_{0}}\right)^{1 / q_{0}} \leq M\left\|f-f_{k}\right\|_{L_{p_{0}}} .
$$

In the same way we establish the convergence of the series $\sum_{|n|>|\alpha|}\left|c_{n} /(n+\alpha \operatorname{sign} n)\right|$.

Consider the case when $\alpha=1 / 2 p_{0}-1$. Then $\exists p^{\prime} \epsilon$ $\left(p_{0}, 2\right): \alpha>1 / 2 p^{\prime}-1$. In this case the system $\left\{e^{i(n+\alpha \operatorname{sign} n) t}\right\}_{n \neq 0}$ is minimal in $L_{p^{\prime}}$, and, consequently, from the results of the paper [16] it holds the Hausdorff-Young inequality; that is,

$$
\left(\sum_{n \neq 0}\left|c_{n}\right|^{q^{\prime}}\right)^{1 / q^{\prime}} \leq M\|f\|_{L_{p^{\prime}}},
$$

where $1 / p^{\prime}+1 / q^{\prime}=1$.

From the previous reasonings we get the absolute convergence of the series $\sum_{|n|>|\alpha|}\left|c_{n} /(n+\alpha \operatorname{sign} n)\right|$. The lemma is proved.
Theorem 4. Let $p \in(1,+\infty), \rho(t)=\prod_{i=1}^{l}\left(\sin \left|\left(t-\tau_{i}\right) / 2\right|\right)^{\beta_{i}}$ be a weight function, $\alpha(t) \equiv \alpha t, \beta(t) \equiv \alpha t, \alpha \in R$ be a real parameter, and the inequalities $-1<\beta_{i}<p / q, i=\overline{1, l} ; 1 / 2 p-$ $1 / 2>\alpha>1 / 2 p-1$ hold. Then the following statements are equivalent:

(1) system (2) forms a basis for $W_{p, \rho}(-\pi, \pi)$;

(2) system (1) forms a basis for $L_{p, \rho}(-\pi, \pi)$.

Proof. At first, suppose that the system (1) forms a basis for $L_{p, \rho}(-\pi, \pi)$. Let us show that the system $\left\{\widehat{u}_{n}\right\}_{n=0}^{\infty}$ forms a basis for $\mathscr{L}_{p, \rho}$, where $\widehat{u}_{0}=(0 ; 1), \widehat{u}_{n}=(i(n+$ $\left.\alpha \operatorname{sign} n) e^{i(n+\alpha \operatorname{sign} n) t} ; e^{-i(n+\alpha \operatorname{sign} n) \pi}\right), n \neq 0$.

It is enough to prove that the arbitrary element $\widehat{u}=(u ; \lambda)$ of $\mathscr{L}_{p, \rho}$ has the unique expansion

$$
\widehat{u}=\sum_{n \neq 0} \lambda_{n} \widehat{u}_{n}
$$

that is,

$$
\begin{gathered}
u(t)=\sum_{n \neq 0} i(n+\alpha \operatorname{sign} n) \lambda_{n} e^{i(n+\alpha \operatorname{sign} n) t}, \\
\lambda=\lambda_{0}+\sum_{n \neq 0} \lambda_{n} .
\end{gathered}
$$

Since the system (1) forms a basis for $L_{p, \rho}(-\pi, \pi)$, then the expansion (23) holds and the coefficients $\lambda_{n}(n \neq 0)$ are uniquely determined. By Lemma 3 the series $\sum_{n \neq 0} \lambda_{n}$ absolutely converges. Then it is clear that the number $\lambda_{0}$ from (24) is uniquely determined. This means that the system $\left\{\widehat{u}_{n}\right\}_{n=\infty}^{\infty}$ forms a basis for $\mathscr{L}_{p, \rho}$. Consider the system $\left\{v_{n}\right\}_{n=-\infty}^{\infty}$, where $v_{n}=A \widehat{u}_{n}, A[(u, \lambda)]=\lambda+\int_{-\pi}^{t} u(\tau) d t, \widehat{u}=(u ; \lambda) \in L_{p, \rho}$. It is not difficult to see that

$$
v_{0}=1, \quad v_{n}=e^{i(n+\alpha \operatorname{sign} n) t}, \quad n \neq 0 .
$$

Now, let us prove the converse. Assume that the system (2) forms a basis for $W_{p}^{1}(-\pi, \pi)$. Consider the system $\widehat{u}_{n}=A^{-1} v_{n}$, $n \in Z$. It is easy to see that the inverse operator is determined as $A^{-1} v=\left(v^{\prime} ; v(-\pi)\right)$. It is obvious that the system $\left\{\widehat{u}_{n}\right\}_{-\infty}^{\infty}$ forms a basis for $\mathscr{L}_{p, \rho}$. We have

$$
\begin{gathered}
\widehat{u}_{0}=(0 ; 1), \\
\widehat{u}_{n}=\left(i(n+\alpha \operatorname{sign} n) e^{i(n+\alpha \operatorname{sign} n) t} ; e^{-i(n+\alpha \operatorname{sign} n) \pi}\right) .
\end{gathered}
$$

Consequently, $\widehat{u}=(u ; \lambda) \in L_{p, \rho}$ has a unique expansion (22) in $\mathscr{L}_{p, \rho}$. As a result, we obtain that each $u \in L_{p, \rho}$ has a unique expansion of the form (23). Indeed, let there exist another expansion for $u(t)$ in $L_{p, \rho}$ :

$$
u(t)=\sum_{n \neq 0} i(n+\alpha \operatorname{sign} n) \mu_{n} e^{i(n+\alpha \operatorname{sign} n) t} .
$$

The absolute convergence of the series $\sum_{n \neq 0} \mu_{n}$ follows from Lemma 3. Put $\mu_{0}=\lambda-\sum_{n \neq 0} \mu_{n}$. It is clear that the biorthogonal coefficients of the element $\widehat{u}=(u ; \lambda)$ are $\left(\bar{\mu} ; \mu_{0}\right)$, where $\bar{\mu}=\left\{\mu_{n}\right\}_{n \neq 0}$. From the basicity of the system $\left\{\widehat{u}_{n}\right\}_{n=-\infty}^{\infty}$ in $\mathscr{L}_{p, \rho}$ we obtain that $\mu_{n}=\lambda_{n}, n \in Z$. This contradicts our conjecture. The theorem is proved. 


\section{Conflict of Interests}

The authors declare that there is no conflict of interests regarding the publication of this paper.

\section{Acknowledgment}

The authors would like to express their sincere gratitude to Professor Bilal T. Bilalov for his attention to the paper and for valuable advice.

\section{References}

[1] R. E. A. Paley and N. Wiener, Fourier Transforms in the Complex Domain, vol. 19 of American Mathematical Society Colloquium Publications, American Mathematical Society, Providence, RI, USA, 1934.

[2] N. Levinson, Gap and Density Theorems, American Mathematical Society Colloquium Publications, Providence, RI, USA, 1940.

[3] A. M. Sedletskiü, "Biorthogonal expansions of functions in exponential series on intervals of the real axis," Uspekhi Matematicheskikh Nauk, vol. 37, no. 5(227), pp. 51-95, 1982.

[4] E. I. Moiseev, "On basicity of systems of sines and cosines," Doklady Akademii Nauk SSSR, vol. 275, no. 4, pp. 794-798, 1984.

[5] B. T. Bilalov, "The basis property of some systems of exponentials of cosines and sines," Differentsial'nye Uravneniya, vol. 26, no. 1, pp. 10-16, 1990.

[6] B. T. Bilalov, "On the basis property of systems of exponentials, cosines, and sines in $L_{p}$," Doklady. Mathematics, vol. 365, no. 1 , pp. 7-8, 1999.

[7] B. T. Bilalov, "On the basis of some systems of exponentials, cosines and sines in $L_{p}$," Doklady Mathematics, vol. 379, no. 2, pp. 158-160, 2001.

[8] B. T. Bilalov, "Bases of exponentials, sines, and cosines," Differentsial'nye Uravneniya, vol. 39, no. 5, pp. 619-622, 2003.

[9] B. T. Bilalov, "Basis properties of some systems of exponentials, cosines, and sines," Siberian Mathematical Journal, vol. 45, no. 2, pp. 264-273, 2004.

[10] E. I. Moiseev, "Basicity in the weight space of a system of eigen functions of differential operator," Differentsial'nye Uravneniya, vol. 35, no. 2, pp. 200-205, 1999.

[11] Z. A. Kasumov, "On basicity of system of exponents with complex valued coefficients in weighted spaces," in Proceedings of the 2006 International Conference, vol. 1, pp. 165-168, Orel, Russia, October 2006.

[12] D. L. Russell, "On exponential bases for the Sobolev spaces over an interval," Journal of Mathematical Analysis and Applications, vol. 87, no. 2, pp. 528-550, 1982.

[13] E. I. Moiseev, "On differential properties of expansions on the system of sines and cosines," Differentsial'nye Uravneniya, vol. 32, no. 1, pp. 117-126, 143, 1996.

[14] A. M. Sedletskii, "Approximate properties of the system of exponents in Sobolev spaces," Vestnik Moskovskogo Universiteta, no. 6, pp. 3-8, 1999.

[15] S. A. Avdonin and S. A. Ivanov, "Riesz bases of exponentials and divided differences," St. Petersburg Mathematical Journal, vol. 13, no. 3, pp. 339-351, 2002.

[16] B. T. Bilalov and O. K. Karakash, "The Hausdorff-Young type theorem for one system of exponents," Transactions of National Academy of Sciences of Azerbaijan, vol. 24, no. 4, pp. 19-23, 2004. 


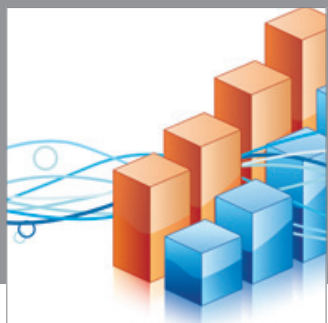

Advances in

Operations Research

mansans

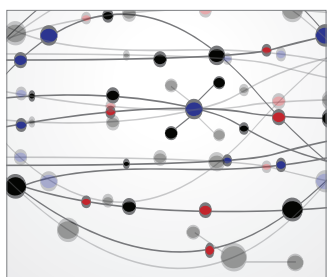

The Scientific World Journal
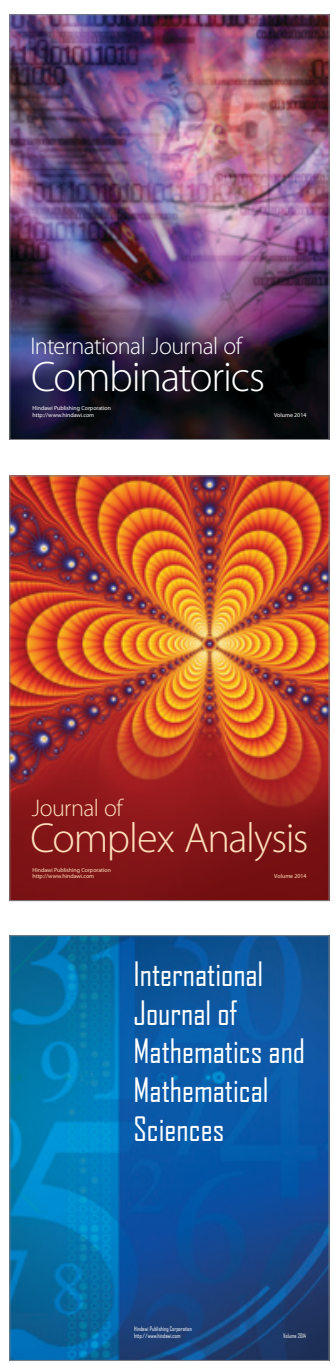
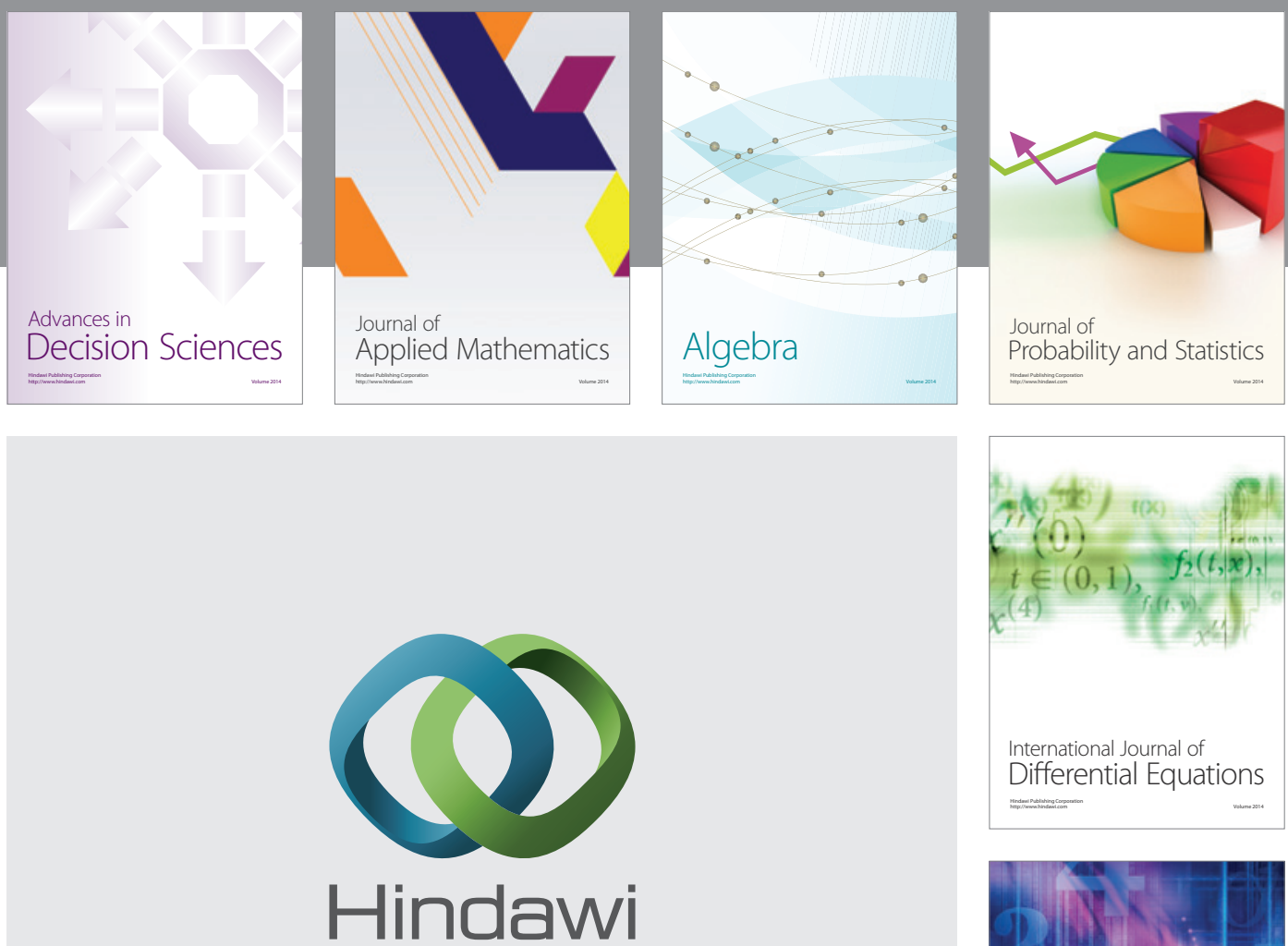

Submit your manuscripts at http://www.hindawi.com
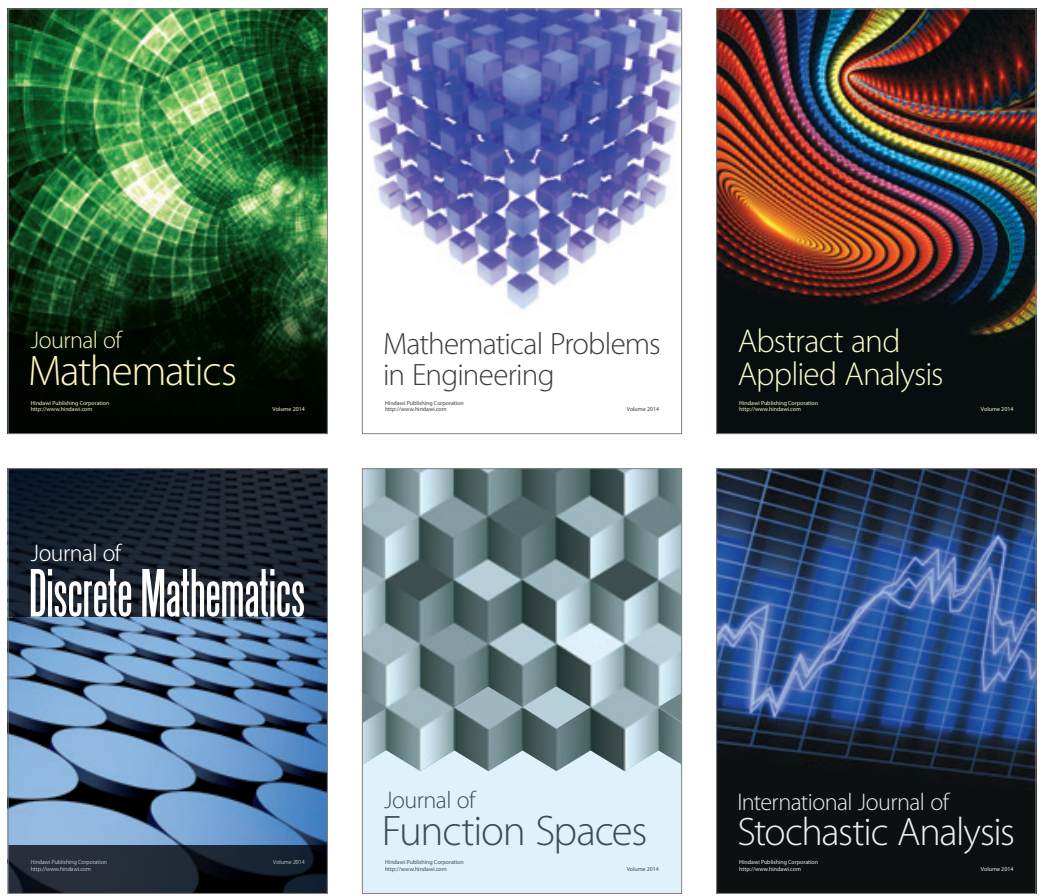

Journal of

Function Spaces

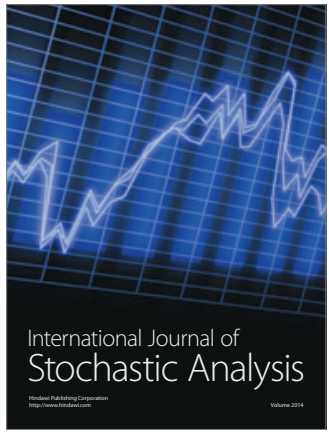

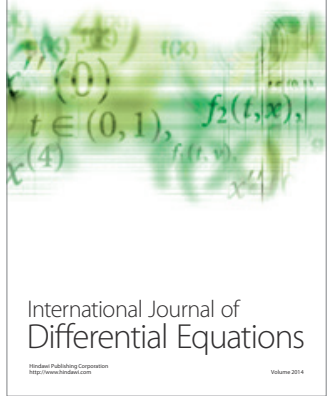
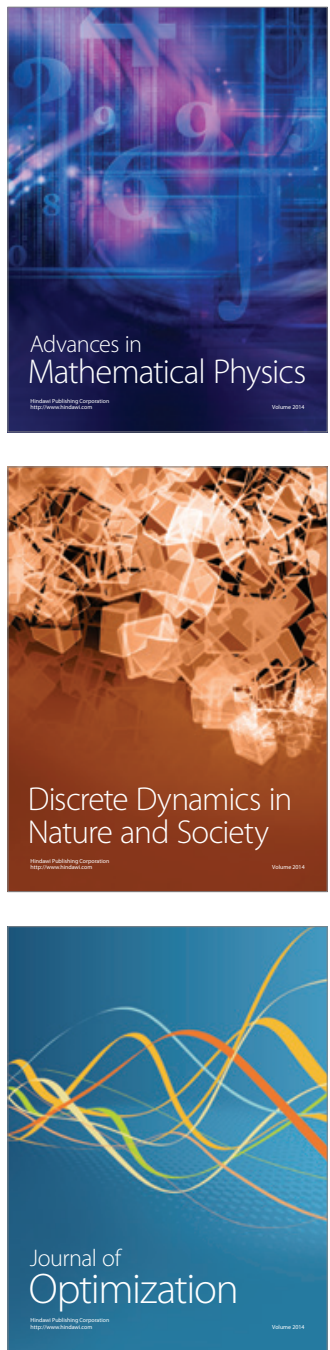